\title{
Zuversichtlich in die Zukunft
}

\section{Michel Matter}

Dr. med., Mitglied des FMH-Zentralvorstandes, Departementsverantwortlicher Dienstleistungen und Berufsentwicklung

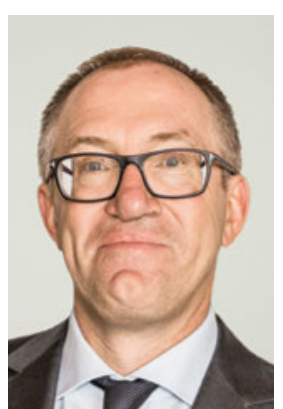

Die Medizin entwickelt sich ständig weiter - und das ist gut so. Allerdings ist das Tempo der digitalen Revolution hoch und stellt die Ärzteschaft vor neue Herausforderungen. Es vergeht kaum ein Monat ohne Konferenz über Digitalisierung, künstliche Intelligenz, Uberisierung der Medizin, Online-Verfolgung von Gesundheitsdaten, Walk-in-Kliniken mit Sofortbehandlung sowie individuell abgestimmte Gesundheitsversorgung dank prädiktiver, partizipativer und präventiver Medizin. Angesichts dieses Wandels müssen wir darauf achten, dass vernünftige Arbeitsbedingungen aufrechterhalten bleiben. Gesundheitsfachpersonen dürfen nicht der Überarbeitung preisgegeben werden, nur weil überzeugende Strategien und Organisationsformen rund um die tägliche Arbeit mit Patienten sowie administrativen Aufgaben fehlen.

Deshalb bedarf die Medizin einer klaren Neuausrichtung. Die Digitalisierung schreitet voran, die Erfassung von Daten (Big Data) breitet sich weiter aus und schafft die Imperien von morgen. Hier muss die FMH ihrer Aufgabe als Vertreterin unseres Berufsstands gerecht werden und die Situation analysieren, die nötigen Massnahmen ergreifen und unsere gemeinsamen Werte verteidigen. Auch wenn es zahlreiche Einzelinteressen und Entwicklungsmöglichkeiten gibt, müssen Lösungen gefunden werden, die auf den gemeinsamen Interessen aufbauen.

\section{Die Medizin bedarf einer klaren Neuausrichtung.}

Die Pharmaindustrie verspricht eine schmerzfreie Medizin dank greifbarer Medikamente. Die Genomik und die Forschung über unser Lebensumfeld, unsere Ernährungsgewohnheiten und unser Verhalten werden eine individuell abgestimmte, prädiktive Gesundheitsversorgung ermöglichen. Zu den Zielen gehören massgeschneiderte klinische und therapeutische Ansätze. Allerdings muss dies im Rahmen eines offenen Austauschs mit sämtlichen patientenbegleitenden Berufsgruppen geschehen. Entscheidend sind dabei die Koordination der Behandlung und die Interprofessionalität.
Die Qualität der medizinischen Behandlung ist mit Entschlossenheit aufrechtzuerhalten, wobei der Mensch weiterhin im Mittelpunkt unseres Tuns stehen muss.

Innovation in der Medizin muss gefördert werden. Die Politik ist aufgefordert, ihre Unterstützung des medizinischen Fortschritts klar zum Ausdruck zu bringen. Ebenso müssen die Ausbildung des ärztlichen Nach-

Wir werden der Ärzteschaft Gehör verschaffen und uns für angemessene Arbeitsbedingungen einsetzen.

wuchses finanziell unterstützt und der Austausch zwischen den Universitäten und den Spitälern verstärkt werden. Zudem soll die Finanzierung von ambulanten und stationären Leistungen vereinheitlicht werden.

2018 wird ein entscheidendes Jahr: Neben der Auferlegung eines neuen Tarifs im ambulanten Bereich durch den Bund, der bis zur Einreichung eines sachgerechten TARMEDs durch die Ärzteschaft (TARCO) und die Tarifpartner gelten wird, gibt es parlamentarische Bestrebungen zur Aufhebung des Kontrahierungszwangs. Dagegen wird sich die FMH engagieren. Ein weiterer Punkt ist das aktuell in der Politik diskutierte Globalbudget, welches eine Rationierung vorsieht. Einen solchen Eingriff, der für die Gesundheitsversorgung fatal wäre, gilt es abzuwenden. Die FMH wird den Anliegen der Ärzteschaft Gehör verschaffen und sich für angemessene Arbeitsbedingungen sowie für eine patientenzentrierte Qualitätsmedizin einsetzen.

Die Leitung des Departements Dienstleistungen und Berufsentwicklung beinhaltet diverse Herausforderungen: z.B. die Definition der Aktivitäten im Austausch mit den übrigen Departementen und die Auseinandersetzung mit der sich wandelnden Medizin. Trotz immer schnellerem Paradigmenwechsel gilt es den Menschen stets in den Mittelpunkt zu stellen. Ich danke Dr. Remo Osterwalder für seine bisher geleistete Arbeit an der Spitze des Departements. Er hat den Weg vorgespurt und das Departement für die Zukunft gut gerüstet. 